\title{
Therapeutics
}

\section{Divalproex did not differ from placebo or lithium in preventing recurrence of new mood episodes in bipolar I disorder, remission phase}

Bowden CL, Calabrese JR, McElroy SL, et al, for the Divalproex Maintenance Study Group. A randomized, placebo-controlled 12-month trial of divalproex and lithium in treatment of outpatients with bipolar I disorder. Arch Gen Psychiatry 2000 May;57:481-9.

\section{QUESTION: In patients with bipolar I disorder in remission, how does divalproex compare with placebo and lithium in preventing recurrence of new mood episodes?}

\section{Design}

1 year randomised \{allocation concealed $*\}$, blinded (clinicians and patients),* controlled trial.

\section{Setting}

37 North American centres.

\section{Patients}

372 outpatients who were $18-75$ years of age, met the DSM-III-R criteria for bipolar disorder, met recovery criteria within 3 months of the onset of an index manic episode, and had $\geqslant 1$ additional episode of mania in the previous 3 years. Other inclusion criteria were scores of $\leqslant 11$ on the Mania Rating Scale (MRS), $\leqslant 13$ on the Depressive Syndrome Scale (DSS), and $\geqslant 60$ on the Global Assessment Scale (GAS). Exclusion criteria were intolerance of divalproex or lithium, recent alcohol or active substance abuse, uncontrolled systemic disorders, seriously suicidal, ongoing psychotherapy, open phase protocol non-adherence, or pregnancy. 369 patients $(99 \%)$ (mean age $39 \mathrm{y}, 51 \%$ women) were included in the intention to treat analysis.

\section{Intervention}

All patients completed a 3 month open phase during which they were treated at the discretion of the investigator. Medications other than divalproex or lithium were discontinued before randomisation. Patients were allocated in a 2:1:1 ratio to divalproex, 71-125 $\mu \mathrm{g} / \mathrm{ml}(\mathrm{n}=187)$; lithium, 0.8-1.2 $\mathrm{mmol} / \mathrm{l}$ $(\mathrm{n}=91)$; or placebo $(\mathrm{n}=94)$.

\section{Main outcome measure}

Time to any mood episode. Secondary outcomes were time to a manic episode, time to a depressive episode, and mean change from baseline on MRS, DSS, and GAS scores.

\section{Main results}

The groups did not differ for time to any mood episode, time to a manic episode, or time to a depressive episode. Mean change from baseline on MRS, DSS, and GAS scores did not differ between groups when analysed using effects for centre. More patients who received divalproex than placebo had tremor $(41 \%$ v $13 \%$, $\mathrm{p}<0.001)$ and weight gain $(21 \% v 7 \%, \mathrm{p}=0.004)$. More divalproex patients had sedation $(42 \% v 35 \%, \mathrm{p}=0.02)$, infection $(27 \% v 13 \%, \mathrm{p}=0.009)$, and tinnitus $(6 \% v 1 \%$, $\mathrm{p}=0.01)$ than lithium group patients. More patients treated with lithium than with divalproex had polyuria $(19 \% v 8 \%, \mathrm{p}=0.01)$, excess thirst $(15 \% v 6 \%, \mathrm{p}=0.01)$, and akathesia $(4 \% v<1 \%, \mathrm{p}=0.04)$.

\section{Conclusion}

In patients with bipolar I disorder in remission, divalproex was no more efficacious than placebo or lithium in preventing recurrence of new mood episodes. *See glossary. $\dagger$ Information provided by author.

\section{COMMENTARY} existed. in maintenance treatment for bipolar disorder. ${ }^{2}$ Psychiatry 2000;57:490-2. term? Eur Neuropsychopharmacol 1999;9(suppl 4):S125-9.
Source of funding: Abbott Laboratories, North Chicago, IL.

For correspondence: Dr C L Bowden Department of Psychiatry, University of Texas Health Science Center at San Antonio, 7703 Floyd Curl Drive, San Antomio, $T X$ 78284-7792, USA. Fax +1210567 3759 .

This important study by Bowden $e t$ al is unique. Despite a strong publication bias in favour of positive results, this study did not show a significant difference between interventions. Furthermore, this is the first placebo controlled maintenance study of pharmacotherapy for bipolar disorder in 25 years and the first ever randomised, blinded, maintenance trial to compare divalproex with placebo and lithium.

Several issues arise when considering why this study showed no difference between the treatments. Firstly, the people in this study probably had bipolar illness of relatively mild severity. They were required to have recovered from a manic episode within the previous 3 months and had 2 consecutive GAS scores $>60$. Nearly one half of the patients had $<10$ previous episodes, 39\% had never required hospital admission, and those with comorbidity or partial responses were excluded from the study. Furthermore, the possibility of being assigned to a placebo control group for up to 1 year with minimal rescue medication may have dissuaded more severely ill people from enrolling in the study. Secondly, the rates of relapse in these patients with relatively mild bipolar disorder were low, thus the difference between relapse rates in each treatment group was less than expected. Furthermore, for many patients the 1 year follow up may have been of insufficient length to capture their next recurrence of any mood episode. Baldessarini et al have suggested using a longer follow up of 2-3 years. ${ }^{1}$ The lower than expected relapse rates, combined with an insufficient sample size, meant that the results did not reach a statistically significant difference although a difference between divalproex and lithium may, and between the active agents and placebo almost certainly, really have

Clinicians await robust, clinically useful evidence to guide bipolar disorder maintenance treatment. A pragmatic, large scale $(n>2000)$, clinically relevant, randomised controlled trial is now being planned that may answer the question as to whether small, but clinically important, differences exist between lithium and divalproex

Robert K Schneider, MD Medical College of Virginia Physicians and Hospitals Virginia Commonwealth University Health System Richmond, Virginia, USA

1 Baldessarini RJ, Tohen M, Tondo L. Maintenance treatment in bipolar disorder. Arch Gen

2 Goodwin GM. Prophylaxis of bipolar disorder: how and who should we treat in the long 